\section{Lyme carditis mimicking giant cell arteritis}

\author{
Krati Chauhan \\ Division of Rheumatology, Department of \\ Internal Medicine, Southern Illinois \\ University, Springfield, IL, USA
}

\begin{abstract}
Presenting an interesting case of a patient who complained of myalgias, fatigue, headache, jaw claudication and scalp tenderness. Patient's physical examination was unremarkable. Laboratory findings showed elevated erythrocyte sedimentation rate and C-reactive protein, bilateral temporal artery biopsy results were negative and first degree atrioventricular block was seen on electrocardiogram. Serology for Borrelia burgdorferi was positive; patient was diagnosed with Lyme carditis and treated with doxycycline. Lyme is a tick-borne, multisystem disease and occasionally its presentation may mimic giant cell arteritis. On followup there was complete resolution of symptoms and electrocardiogram findings.
\end{abstract}

\section{Case Report}

Patient is a 59-year-old male who presented with chief complaint of myalgias. His health changed four months before when he started to develop myalgias. There was no preceding trauma and the symptoms were localized in the shoulder and hip region. Myalgias were non-radiating, associated with morning stiffness for about an hour. He complained of difficulty lifting his arms above his head and getting out of a chair. For the past two weeks the symptoms progressively worsened and he was admitted for further evaluation. Patient complained of intermittent headache localized at the temple region, jaw claudication, scalp tenderness, and of one episode of temperature of $100^{\circ} \mathrm{F}$. He denied vision change and diplopia. Review of symptoms was positive for fatigue present for two months, progressively getting worse and an erythematous rash noticed on left arm six months before. The rash cleared on its own and patient did not seek medical care for it. He denied joint pain, respiratory, cardiovascular, abdominal and genitourinary symptoms.

Past medical history was significant for coronary artery disease for which he underwent stent placement one year before. He was on statin post stent placement, which was discontinued three months before, one month after development of myalgias. Despite discontinuation of statin there was no symptomatic improvement. Other medications included acetylsalicylic acid, clopidogrel and enalapril. He quit smoking after stent placement, prior to that had forty pack year history of smoking, denied alcohol and recreational drug use. He was a farmer form Wisconsin (USA); family history was significant for mother with osteoarthritis of knee. On examination the patient was in no acute distress, vitals were stable. There was no scalp necrosis, no carotid and subclavian artery bruit and bilateral temporal arteries were palpable. Both upper and lower extremity pulses were present and symmetric. Proximal muscle strength could not be tested; patient declined to cooperate because of pain. Distal muscle strength in bilateral upper and lower extremity was within normal limits. Remainder of the physical examination was unremarkable.

Lab evaluation showed normal hemoglobin, platelet, white blood cell counts, liver function, kidney function tests, thyroid stimulating hormone, lactate dehydrogenase, aldolase and creatinine kinase. Erythrocyte sedimentation rate was elevated at $140 \mathrm{~mm} / \mathrm{hr}$ (reference value: 0 -22) and C-reactive protein at $195 \mathrm{mg} / \mathrm{L}$ (reference value: 0-8). Electrocardiogram (EKG) showed first degree atrioventricular (AV) block: PR interval of 222 seconds, which was not present on previous EKG. Patient underwent bilateral temporal artery biopsy for suspicion of temporal arteritis and was started on prednisone $60 \mathrm{mg}$ daily. Blood and urine cultures came back negative; serologic testing for histoplasma, blastomyces and coccidioides was negative. The cerebrospinal fluid (CSF) showed normal white blood cell count, protein and glucose levels. Serum was positive for Borrelia burgdorferi total antibodies detected by enzyme linked immunosorbent assay (ELISA); 2.4, (normal <1); western blot assay showed $5 \operatorname{IgG}(18,23,28,30,39)$ and $3 \operatorname{IgM}(23$, $28,30)$ bands. Diagnosis of Lyme carditis was made based on positive serology for Lyme disease and EKG findings showing first degree heart block. The patient was started on doxycycline $100 \mathrm{mg}$ twice daily for a three-week course. Bilateral temporal artery biopsy results came back negative, fundus exam was unremarkable and prednisone was discontinued. On follow-up appointment he had marked improvement of symptoms, physical examination including proximal muscle strength in bilateral upper and lower extremity was normal. Repeat EKG showed resolution of AV block; ESR and CRP returned to normal limits.

\section{Discussion}

Here we present a case of Lyme carditis
Correspondence: Krati Chauhan, Division of Rheumatology, Department of Internal Medicine, Southern Illinois University, $751 \mathrm{~N}$ Rutledge St, Suite 2300, Springfield, 62701 IL, USA.

Tel.: +1.217.545.0184 - Fax: +1.217.545.4485.

E-mail: kchauhan85@siumed.edu

Key words: Lyme carditis; giant cell arteritis; Borrelia burgdorferi.

Conflict of interest: the authors declare no potential conflict of interest.

Received for publication: 17 May 2015.

Revision received: 11 July 2015.

Accepted for publication: 13 July 2015.

This work is licensed under a Creative Commons Attribution NonCommercial 3.0 License (CC BYNC 3.0).

(C) Copyright K.Chauhan, 2015

Licensee PAGEPress, Italy

Rheumatology Reports 2015; 7:6023

doi:10.4081/rr.2015.6023

mimicking giant cell arteritis (GCA).

Carditis is a manifestation of disseminated Lyme disease, presenting within weeks or months after a bite from a Ioxedes scapularis tick, inoculating Borrelia burgdorferi into the human host. ${ }^{1,2}$ Atrioventricular conduction disturbances are the most common manifestation of Lyme carditis..$^{1,2}$ As in our patient, outpatient management with oral antibiotics is appropriate for patients with first degree AV block with PR interval less than $300 \mathrm{msec}$, since these patients are at low risk of progression to complete heart block. ${ }^{3}$ Most of the conduction abnormalities have a benign prognosis and resolve after initiation of antibiotic therapy. ${ }^{4}$

There have been previous cases of Lyme disease initially diagnosed as GCA. Pizzarello et $a l .,{ }^{5}$ presented a case of 71 year old male who presented with left eye vision loss and headache. Left eye exam showed visual acuity of 20/50 and optic nerve swelling. He was diagnosed with ischemic optic neuropathy related to GCA and started on $80 \mathrm{mg}$ of prednisone per day. However his visual acuity deteriorated to perception of hand motion. Serology and culture for Lyme disease were obtained because he was from an endemic region. Serology was equivocal [indirect immunofluorescent (IFA) titer, 1:64] and blood culture grew Borrelia species, different from burgdorferi. Lyme disease was diagnosed and patient was given a course of IV ceftriaxone, resulting in improvement of vision in left eye to finger counting.

Traisk et al. ${ }^{6}$ described three cases initially treated as GCA, subsequently diagnosed as Lyme neuroborreliosis. All three were female 
patients aged 76, 72 and 75 . The first patient had an established diagnosis of GCA and had been taking $15 \mathrm{mg}$ of prednisone and tocilizumab. The second patient had a diagnosis of polymyalgia rheumatica and had been off prednisone for the past three weeks. The third patient did not have a prior rheumatologic diagnosis. All three presented with visual deterioration and headaches, examination revealed swollen optic discs. These patients were started on IV corticosteroid, with no improvement of visual findings. CSF examination revealed pleocytosis and intrathecal antibody production to $B$. burgdorferi leading to a diagnosis of Lyme neuroborreliosis. All three received antibiotic therapy with resolution of optic disc edema and improvement of visual acuity. Our case differs from the above mentioned cases: our patient, at 59 years old, is younger than those four patients, who were in their 70s and had significantly elevated markers of inflammation (ESR and CRP). Effectively, ESR (normal $<30 \mathrm{~mm} / \mathrm{hr}$ ) was 38 $\mathrm{mm} / \mathrm{hr}$ for the case of Pizzarello et al., ${ }^{5}$ and 5.35 and $30 \mathrm{~mm} / \mathrm{hr}$ for the 2 cases described by Traisk et al. ${ }^{6}$ Moreover, Pizzarello et al. ${ }^{5}$ did not report CRP, while Traisk et al. ${ }^{6}$ reported CRP $<1 \mathrm{mg} / \mathrm{L}$ and $<2 \mathrm{mg} / \mathrm{L}$ for two patients, while the third one had a marginal elevation at 8 $\mathrm{mg} / \mathrm{L}$ (normal range: $<8 \mathrm{mg} / \mathrm{L}){ }^{6}$

There is no reference of cardiac involvement either by Pizzarello et al..$^{5}$ or by Traisk $e t$ $a l .{ }^{6}$ in contrast to our patient who had Lyme carditis manifested by AV block. CSF findings in our patient were within normal limits whereas all three patients of Traisk et al. had CSF pleocytosis and intrathecal antibody production to Borrelia burgdorferi; there is no mention about CSF examination by Pizzarello et al..$^{5}$

Four patients of polymyalgia rheumatica presenting with proximal pain and stiffness who were subsequently diagnosed as Lyme disease based on serology were described by Schwartzberg et al. ${ }^{7}$ and one case by Charkravarty et al. ${ }^{8}$ However, unlike Pizzarello et al., ${ }^{5}$ Traisk et al. ${ }^{6}$ and our case there was no mention of visual complaints, optic exam findings and headaches suggestive of GCA. Also there is no discussion of cardiac manifestations, again contrasting with our patient.

\section{Conclusions}

This is a case of Lyme disease with cardiac involvement portraying as giant cell arteritis. Lyme is a multisystem disease involving skin, heart, joints and nervous system. In regions where Lyme disease is endemic it should be considered in differential diagnosis of giant cell arteritis and polymyalgia rheumatica.

\section{References}

1. van der Linde MR. Lyme carditis: clinical characteristics of 105 cases. Scand J Infect Dis Suppl 1991;77:81-4.

2. Steere AC, Batsford WP, Weinberg M, et al. Lyme carditis: cardiac abnormalities of Lyme disease. Ann Intern Med 1980;93:816.

3. Costello JM, Alexander ME, Greco KM, et al. Lyme carditis in children: presentation, predictive factors, and clinical course. Pediatrics 2009;123:e835-41.

4. Lelovas P, Dontas I, Bassiakou E, Xanthos T. Cardiac implications of Lyme disease, diagnosis and therapeutic approach. Int $\mathbf{J}$ Cardiol 2008;129:15-21.

5. Pizzarello LD, MacDonald AB, Semlear R, et al. Temporal arteritis associated with Borrelia infection. A case report. J Clin Neuroophthalmol 1989;9:3-6.

6. Traisk F, Andersson M, Svenungsson E. Three cases of neuroborreliosis misdiagnosed as giant cell arteritis. Scand J Rheumatol 2012;41:158-60.

7. Schwartzberg M, Weber CA, Musico J. Lyme borreliosis presenting as a polymyalgia rheumatica-like syndrome. $\mathrm{Br} \mathrm{J}$ Rheumatol 1995;34:392-3.

8. Chakravarty K, Merry P. Polymyalgia rheumatica-a delayed sequelae of Borrelia infection? Br J Rheumatol 1992;31:647-8. 